\section{Ventral Tegmental Area of Midbrain}

Michelle L. Block

Stark Neuroscience Research Institute, Indiana

University, Indianapolis, IN, USA

\section{Synonyms}

Ventral tegmental area

\section{Definition}

The ventral tegmental area (VTA) is comprised of multiple nuclei and is located in the mesencephalon, dorsomedial to the substantia nigra and ventral to the red nucleus. The VTA is comprised of dopamine, glutamate, and GABA neurons and is an essential component of both the mesolimbic pathway and the mesocortical pathway.

\section{Current Knowledge}

The mesolimbic dopamine projection from the VTA to the nucleus accumbens has been implicated in motivation, emotion, the positive symptoms of schizophrenia, and the rewarding effects of drugs of abuse. The dopaminergic neurons in the mesocortical pathway, which originates from the VTA and projects to the cortex, are associated with motivation, attention, planning, social behavior, and the negative symptoms of schizophrenia. For more detailed information, see Ikemoto (2007).

\section{Cross-References}

Limbic System

- Nucleus Accumbens

\section{References and Reading}

Ikemoto, S. (2007). Dopamine reward circuitry: Two projection systems from the ventral midbrain to the nucleus accumbens-olfactory tubercle complex. Brain Research Reviews, 56(1), 27-78. 